Open Access

\title{
Effects of dietary protein/energy ratio on growth performance, carcass trait, meat quality, and plasma metabolites in pigs of different genotypes
}

Yingying Liu ${ }^{1,2,5 \dagger}$, Xiangfeng Kong ${ }^{1 \dagger}$, Guoli Jiang ${ }^{1,5}$, Bi'e Tan $^{1}$, Jinping Deng ${ }^{3}$, Xiaojian Yang ${ }^{4}$, Fengna Li ${ }^{1^{*}}$, Xia Xiong ${ }^{1}$ and Yulong Yin ${ }^{1 *}$

\begin{abstract}
Background: The protein/energy ratio is important for the production performance and utilization of available feed resources by animals. Increased protein consumption by mammals leads to elevated feed costs and increased nitrogen release into the environment. This study aimed to evaluate the effects of dietary protein/energy ratio on the growth performance, carcass traits, meat quality, and plasma metabolites of pigs of different genotypes.

Methods: Bama mini-pigs and Landrace pigs were randomly assigned to two dietary treatment groups (Chinese conventional diet with low protein/energy ratio or National Research Council diet with high protein/energy ratio; $\mathrm{n}=24$ per treatment) in a $2 \times 2$ factorial arrangement. Blood and muscle samples were collected at the end of the nursery, growing, and finishing phases.

Results: We observed significant interactions $(P<0.05)$ between breed and diet for total fat percentage, intramuscular fat (IMF) content, protein content in biceps femoris (BF) muscle, and plasma urea nitrogen (UN) concentration in the nursery phase; for average daily gain (ADG), average daily feed intake (ADFI), dry matter, IMF content in psoas major (PM) muscle, and plasma total protein and albumin concentrations in the growing phase; and for drip loss and plasma UN concentration in the finishing phase. Breed influenced $(P<0.05)$ growth performance, carcass traits, and meat quality, but not plasma metabolites. Throughout the trial, Landrace pigs showed significantly higher $(P<0.05) A D G$, ADFI, dressing percentage, lean mass rate, and loin-eye area than did Bama mini-pigs, but significantly lower $(P<0.05)$ feed/gain ratio, fat percentage, backfat thickness, and IMF content. Dietary protein/energy ratio influenced the $\mathrm{pH}$ value, chemical composition of BF and PM muscles, and plasma activities of glutamic-pyruvic transaminase and gamma-glutamyl transpeptidase, and plasma concentration of UN.
\end{abstract}

Conclusions: Compared with Landrace pigs, Bama mini-pigs showed slower growth and lower carcass performance, but had better meat quality. Moreover, unlike Landrace pigs, the dietary protein/energy ratio did not affect the growth performance of Bama mini-pigs. These results suggest that, in swine production, low dietary protein/energy ratio may be useful for reducing feed costs and minimizing the adverse effects of ammonia release into the environment.

Keywords: Dietary protein/energy ratio, Growth performance, Meat quality, Mini-pig, Plasma metabolites

\footnotetext{
*Correspondence: lifengna@isa.ac.cn; yinyulong@isa.ac.cn

${ }^{\dagger}$ Equal contributors

'Key Laboratory of Agro-ecological Processes in Subtropical Region, Hunan Provincial Engineering Research Center of Healthy Livestock and Poultry, and Scientific Observing and Experimental Station of Animal Nutrition and Feed Science in South-Central, Ministry of Agriculture, Institute of Subtropical Agriculture, Chinese Academy of Sciences, Changsha, Hunan 410125, China

Full list of author information is available at the end of the article
} 


\section{Background}

The pig is one of the most economically important species among domesticated livestock and is a major protein source for human consumption. A major objective of pig production is to increase skeletal muscle growth and reduce excess fat accretion. Livestock producers use nutritional modifiers in an attempt to increase protein accretion in the muscle, while they often simultaneously reduce fat deposition [1, 2]. The pattern of fat deposition in pigs in the growingfinishing phase affects carcass and meat quality [3]. Genetic selection of pigs for leaner meat has also resulted in reduced intramuscular fat (IMF) content, and consumers perceive the meat thus obtained as tougher, less moist, and poorly flavored. Thus, the livestock industry faces the challenge of increasing the IMF content of pork so that consumers may have a satisfactory experience, while simultaneously producing minimal visible fat, which is a deterrent to health-conscious consumers.

In China, several rich resources of indigenous pig breeds are available, with more than 30 breeds established. In addition to their economic significance, pigs, particularly miniature pigs (mini-pigs), are considered important animal models for human disease and xenotransplantation research because of their physiological and anatomical similarities to humans [4, 5]. Bama mini-pigs (Sus scrofa domestica), a Chinese indigenous mini-pig breed which originated in Bama County, Guangxi Province, is a promising animal model $[6,7]$ with an obese genotype. In contrast, Landrace, a representative lean genotype, is fastgrowing breed and produces a relatively large amount of meat, and it is therefore more attractive to producers. Although muscle growth and meat quality considerably differ between Western and indigenous Chinese pig breeds, it is not clear how nutrients mediate the effect of genetic background on animal growth and meat quality. The growth and development processes of pigs, which involve changes in body weight $(\mathrm{BW})$ and shape as well as metabolic and physiological functions, depend on factors such as the breed, nutritional status, and feeding condition of the animal. Against this background, the objective of the present study was to examine the effects of dietary protein/energy ratio on the growth performance, muscle development, and plasma metabolites (which are indicators of nitrogen metabolism) in Bama mini-pigs and Landrace pigs at different phases of growth.

\section{Methods}

\section{Study animals}

The experiment was carried out in accordance with the Chinese guidelines for animal welfare and experimental protocol, and was approved by the Animal Care and Use Committee of the Institute of Subtropical Agriculture,
Chinese Academy of Sciences [8]. Ninety-six barrows (including 48 Bama mini-pigs, a Chinese local breed [average initial BW, $3.38 \pm 0.96 \mathrm{~kg}$ ], and 48 Landrace piglets [average initial BW, $7.68 \pm 0.89 \mathrm{~kg}$ ]) were used in this study.

\section{Study design}

The experiment was a $2 \times 2$ factorial arrangement, with two breeds (Bama mini-pig vs. Landrace) and two dietary treatments (Chinese conventional diet [GB diet] and National Research Council [NRC] diet), resulting in a total of four treatments (Table 1). Piglets of each breed were randomly assigned to one of two dietary treatment groups ( $\mathrm{n}=24$ per treatment). The NRC diet was formulated to meet the nutrient requirements recommended by NRC (2012) [9] and had a high protein/energy ratio, whereas the GB diet was formulated per the recommendations of Chinese National Feeding Standard for Swine (GB, 2004) [10] and had a low protein/energy ratio (Table 2 and Additional file 1: Table S1). The animals were individually housed in $0.6 \mathrm{~m} \times 1.2 \mathrm{~m}$ pens with hard plastic slatted flooring. Each pen was equipped with a stainless steel feeder and a nipple drinker. The animals had free access to drinking water and feed. The room temperature was maintained at $25-27{ }^{\circ} \mathrm{C}$. All pigs were fed three times per day at 0800, 1300, and $1800 \mathrm{~h}$. Dietary phase changes were noted on the day on which the pigs were weighed; these changes were noted on the same day for all treatment types. Every 2 weeks, feed intake was recorded in order to determine average daily gain (ADG), average daily feed intake (ADFI), and the feed intake to body gain ratio (F/G).

\section{Sample collection}

According to the feeding standards, the BW range for nursery, growing, and finishing phases (ending with market weight) was defined as 8-20, 20-50, and 50-90 kg, respectively, for Landrace pigs, and 3-15, 15-35, and 35-55 kg, respectively, for Bama mini-pig (Table 1). Nursery, growing, and finishing phases comprised feeding for 39, 44 and 30 days, respectively, for Landrace pigs, and 55, 48 and 21 days, respectively, for Bama mini-pigs. At the end of each phase (i.e., when BW reached 20, 50, and $90 \mathrm{~kg}$ for Landrace pigs, and 15, 35, and $55 \mathrm{~kg}$ for Bama mini-pigs), eight pigs from each treatment were randomly weighed, bled, and sacrificed for the evaluation of carcass characteristics and meat quality. Briefly, the animals were fasted for $12 \mathrm{~h}$ to avoid the effect of feed intake on postprandial biochemical measurements and then pre-slaughter BW was measured. Thereafter, blood samples were collected into $10-\mathrm{mL}$ centrifuge tubes containing sodium heparin (14.3 USP units/mL). Next, the pigs were held under general anesthesia and sacrificed by a 
Table 1 Animals and treatments

\begin{tabular}{|c|c|c|c|c|c|c|}
\hline \multirow[t]{2}{*}{ Item } & \multicolumn{3}{|c|}{ Landrace pig } & \multicolumn{3}{|c|}{ Bama mini-pig } \\
\hline & Body weight & GB diet group & NRC diet group & Body weight & GB diet group & NRC diet group \\
\hline Nursery phase & $10-20, \mathrm{~kg}$ & GB diet 1 & NRC diet 1 & $8-15, \mathrm{~kg}$ & GB diet 1 & NRC diet 1 \\
\hline Growing phase & $20-50, \mathrm{~kg}$ & GB diet 2 & NRC diet 2 & $15-35, \mathrm{~kg}$ & GB diet 2 & NRC diet 2 \\
\hline Finishing phase & $50-90, \mathrm{~kg}$ & GB diet 3 & NRC diet 3 & $35-55, \mathrm{~kg}$ & GB diet 3 & NRC diet 3 \\
\hline
\end{tabular}

GB diet, Chinese conventional diet

jugular vein injection of $4 \%$ sodium pentobarbital solution (40 mg/kg BW) [11]. After the head, legs, tail, and viscera were removed, the carcass was split longitudinally. Longissimus dorsi (LD), biceps femoris (BF), and psoas major (PM) muscles from the rightside of each carcass were immediately sampled after slaughter, and stored at $-20{ }^{\circ} \mathrm{C}$ for biochemical analysis (including evaluation of dry matter [DM], crude protein $[\mathrm{CP}]$, and crude lipid contents). The LD muscle on the right-side carcass was removed, and approximately $2.5-\mathrm{cm}$-thick sections were cut from the anterior end for assessment of meat quality before and after chilling the carcass for 24 $\mathrm{h}$ [2]. Blood samples were subsequently centrifuged at $900 \times g$ for $10 \mathrm{~min}$ at $4{ }^{\circ} \mathrm{C}$ to recover plasma. Plasma samples were stored at $-80{ }^{\circ} \mathrm{C}$ until analysis.

\section{Determination of carcass composition}

Pre-slaughter BW, carcass weight, carcass length, backfat thickness, and loin-eye area at the $10^{\text {th }}$ rib were measured immediately post-mortem according to the Chinese Guidelines on Performance Measurement Technology and Regulations for Pigs [12]. Carcass straight length was measured from the first rib to the end of the pubic bone. Backfat thickness was measured using a vernier caliper, and the average measurements at three points: the first rib, last rib, and last lumbar vertebra were recorded. The left side of each carcass was weighed and then physically dissected into skin, skeletal muscle, fat, and bone for evaluation of carcass characteristics. These components were weighed, and the weights were multiplied by 2 to calculate the percentage of the whole carcass that each component constituted. Dressing percentage was calculated as carcass weight divided by live BW.

Table 2 Nutrient content of experimental diets

\begin{tabular}{|c|c|c|c|c|c|c|}
\hline Item & $\begin{array}{l}\text { NRC } \\
\text { diet } 1\end{array}$ & $\begin{array}{l}\text { NRC } \\
\text { diet } 2\end{array}$ & $\begin{array}{l}\text { NRC } \\
\text { diet } 3\end{array}$ & $\begin{array}{l}\text { GB } \\
\text { diet } 1\end{array}$ & $\begin{array}{l}\text { GB } \\
\text { diet } 2\end{array}$ & $\begin{array}{l}\text { GB } \\
\text { diet } 3\end{array}$ \\
\hline $\begin{array}{l}\text { Digestible energy, } \\
\mathrm{MJ} / \mathrm{kg}\end{array}$ & 14.22 & 14.21 & 14.22 & 13.46 & 13.40 & 13.40 \\
\hline Crude protein, \% & 20.06 & 18.01 & 15.11 & 18.03 & 16.05 & 13.46 \\
\hline $\begin{array}{l}\text { Protein/energy } \\
\text { ratio }\end{array}$ & 1.41 & 1.27 & 1.06 & 1.34 & 1.20 & 1.00 \\
\hline
\end{tabular}

GB diet, Chinese conventional diet

\section{Assessment of meat quality}

Meat quality was examined at the end of experiment by determining the $\mathrm{pH}$, muscle color, drip loss, and cooking yield. Initial $\mathrm{pH}\left(\mathrm{pH}_{45 \mathrm{~min}}\right)$ and final $\mathrm{pH}\left(\mathrm{pH}_{24 \mathrm{~h}}\right)$ values were measured in triplicate at the $6^{\text {th }}$ to $7^{\text {th }}$ rib position at $45 \mathrm{~min}$ and 24-h postmortem, respectively, using a hand-held pH meter (Russell CD700; Russell pH Limited, Germany). Muscle color scores were assigned to LD muscle at the $10^{\text {th }}$ rib interface by using a Konica Minolta chromameter (CR410; Konica Minolta Sensing, Inc., Tokyo, Japan) with an 8-mm measuring port, D65 illuminant, and 10 observers. Hunter lightness ( $\left.\mathrm{L}^{*}\right)$, redness $\left(a^{\prime \prime}\right)$, and yellowness $\left(b^{\prime \prime}\right)$ values were recorded in triplicate. For evaluating drip loss, on the day of slaughter, approximately $100 \mathrm{~g}$ fresh LD muscle was weighed and placed in a Whirl-Pak bag, suspended in a $4{ }^{\circ} \mathrm{C}$ cooler for $24 \mathrm{~h}$, reweighed, and drip loss was recorded. Percentage of cooking meat was measured by determining the weight of cooked LD muscle. The muscle sample was weighed and covered in a container before cooking. Immediately after cooking for $45 \mathrm{~min}$ at $100{ }^{\circ} \mathrm{C}$, the sample was removed from the container and dried with a paper towel, then reweighed. Cooking yield was expressed using the following formula: Cooking yield $=($ cooked weight/raw weight) $\times 100$.

\section{Chemical analysis of skeletal muscle}

Chemical composition of the skeletal muscle was analyzed in duplicate according to AOAC methods (1997) [13]. DM content of muscle was determined gravimetrically by oven drying the samples at $110{ }^{\circ} \mathrm{C}$ for $24 \mathrm{~h}$. CP and lipid contents were measured using Kjeldahl and Soxhlet extraction methods, respectively [2].

\section{Analysis of plasma metabolites}

Plasma activities of alkaline phosphatase (ALP), glutamicpyruvic transaminase (GPT), glutamic-oxaloacetic transaminase (GOT), lactate dehydrogenase (LDH), creatine phosphokinase $(\mathrm{CPK})$, and gamma-glutamyl transpeptidase (GGT), and plasma concentrations of albumin (Alb), total protein (TP), ammonia (AMM), and urea nitrogen (UN) were analyzed using a CX-4 Automatic Biochemical Analyzer (Beckman Inc., USA) and commercial kits 
(Leadman Biochemistry Technology Company, Beijing, China) according to the manufacturers' instructions [14].

\section{Statistical analysis}

Data were analyzed by a mixed-effects model using the SAS version 8.2 (SAS Institute Inc., Cary, NC, USA). Diet, breed, and their interactions were included in the statistical model. Effects were considered statistically significant at $P<0.05$. Probability values between 0.05 and 0.10 were considered to be trends.

\section{Results}

\section{Growth performance}

All pigs showed healthy growth throughout the experimental period. ADG and ADFI of Bama mini-pigs were lower $(P<0.05)$, whereas $\mathrm{F} / \mathrm{G}$ was higher $(P<0.05)$ when compared with Landrace pigs in the same phase and fed the same diet (Table 3). The growth performance of Bama mini-pigs did not significantly differ $(P>0.05)$ between dietary treatments in any of the three phases. However, in the growing phase, Landrace pigs fed the GB diet had higher $(P<0.05)$ ADG and ADFI than those fed the NRC diet, indicating breed $\times$ diet interactions $(P<0.05)$. In contrast, in the finishing phase, Landrace pigs fed the GB diet had lower $(P<0.05)$ ADG than those fed the NRC diet.

\section{Carcass quality}

Table 4 shows the effects of treatments on carcass characteristics. In all phases, Bama mini-pigs had significantly lower $(P<0.05)$ dressing percentage, carcass length, loineye area, and skeletal muscle percentage (15-25 units difference), but higher $(P<0.05)$ total fat percentage $(1-20$ units difference) than did Landrace pigs. Except during the nursery phase, the backfat thickness of Bama minipigs was greater $(P<0.05)$ than that of Landrace pigs (2- to 5-fold difference after the nursery phase). In the nursery and finishing phases, Bama mini-pigs fed the NRC diet showed significantly higher $(P<0.05)$ dressing percentages than those fed the GB diet. In the nursery phase, Landrace pigs fed the NRC diet had significantly greater $(P<0.05)$ loin-eye area and reduced backfat thickness $(P<0.05)$ than those fed the GB diet. However, in the growing phase, Landrace pigs fed the GB diet had longer carcasses, more total skeletal muscle, and lower percentage of fat than those fed the NRC diet $(P<0.05)$.

\section{Meat quality}

Compared with Landrace pigs, Bama mini-pigs had lower $(P<0.05) \mathrm{pH}_{45 \mathrm{~min}}$ and $\mathrm{pH}_{24 \mathrm{~h}}$, but higher $(P<0.05)$ $\mathrm{a}^{*}$ and cooking yield (Table 5$)$. For Bama mini-pigs, the NRC diet showed increased $(P<0.05) \mathrm{pH}_{24 \mathrm{~h}}$ in the finishing phase. A breed $\times$ diet interaction $(P<0.05)$ was observed for dripping loss.

Table 3 Effects of dietary protein/energy ratio and breed on growth performance of pigs

\begin{tabular}{|c|c|c|c|c|c|c|c|c|}
\hline \multirow[t]{2}{*}{ Item } & \multicolumn{2}{|c|}{ Landrace pig } & \multicolumn{2}{|c|}{ Bama mini-pig } & \multirow[t]{2}{*}{ SEM } & \multicolumn{3}{|c|}{$P$-value } \\
\hline & GB diets & NRC diets & GB diets & NRC diets & & Breed & Diet & $B \times D$ \\
\hline \multicolumn{9}{|l|}{ Nursery phase $(n=24)$} \\
\hline Initial BW, kg & $7.71^{\mathrm{a}}$ & $7.64^{\mathrm{a}}$ & $3.37^{\mathrm{b}}$ & $3.38^{\mathrm{b}}$ & 0.19 & $<0.001$ & 0.88 & 0.83 \\
\hline Final BW, kg & $22.95^{\mathrm{a}}$ & $23.06^{\mathrm{a}}$ & $17.29^{b}$ & $17.91^{\mathrm{b}}$ & 0.81 & $<0.001$ & 0.66 & 0.76 \\
\hline Average daily gain, $\mathrm{g}$ & $390.80^{a}$ & $395.40^{\mathrm{a}}$ & $253.10^{\mathrm{b}}$ & $264.10^{b}$ & 15.36 & $<0.001$ & 0.62 & 0.84 \\
\hline Average daily feed intake, $\mathrm{kg}$ & $0.76^{\mathrm{a}}$ & $0.71^{\mathrm{a}}$ & $0.62^{\mathrm{b}}$ & $0.63^{b}$ & 0.03 & $<0.001$ & 0.42 & 0.41 \\
\hline Feed to gain ratio & $1.97^{\mathrm{b}}$ & $1.87^{\mathrm{b}}$ & $2.53^{\mathrm{a}}$ & $2.38^{\mathrm{a}}$ & 0.07 & $<0.001$ & 0.09 & 0.73 \\
\hline \multicolumn{9}{|l|}{ Growing phase $(n=16)$} \\
\hline Initial BW, kg & $24.74^{\mathrm{a}}$ & $25.15^{\mathrm{a}}$ & $20.55^{b}$ & $19.66^{\mathrm{b}}$ & 0.66 & $<0.001$ & 0.73 & 0.34 \\
\hline Final BW, kg & $61.82^{\mathrm{a}}$ & $56.37^{b}$ & $38.81^{c}$ & $38.95^{\mathrm{c}}$ & 1.70 & $<0.001$ & 0.13 & 0.12 \\
\hline Average daily gain, $\mathrm{g}$ & $842.80^{a}$ & $709.60^{\mathrm{b}}$ & $380.60^{c}$ & $401.80^{c}$ & 31.39 & $<0.001$ & 0.09 & 0.02 \\
\hline Average daily feed intake, $\mathrm{kg}$ & $1.96^{\mathrm{a}}$ & $1.64^{\mathrm{b}}$ & $1.79^{\mathrm{b}}$ & $1.68^{b}$ & 0.05 & 0.23 & $<0.001$ & 0.04 \\
\hline Feed to gain ratio & $2.33^{\mathrm{a}}$ & $2.43^{b}$ & $4.77^{\mathrm{a}}$ & $4.65^{a}$ & 0.27 & $<0.001$ & 0.98 & 0.70 \\
\hline \multicolumn{9}{|l|}{ Finishing phase $(n=8)$} \\
\hline Initial BW, kg & $65.03^{\mathrm{a}}$ & $62.83^{\mathrm{a}}$ & $44.07^{b}$ & $45.82^{\mathrm{b}}$ & 1.07 & $<0.001$ & 0.87 & 0.15 \\
\hline Final BW, kg & $91.03^{\mathrm{a}}$ & $92.03^{\mathrm{a}}$ & $51.40^{\mathrm{b}}$ & $52.76^{\mathrm{b}}$ & 1.46 & $<0.001$ & 0.52 & 0.92 \\
\hline Average daily gain, $\mathrm{g}$ & $866.70^{b}$ & $973.30^{\mathrm{a}}$ & $348.80^{c}$ & $330.50^{c}$ & 41.87 & $<0.001$ & 0.40 & 0.24 \\
\hline Average daily feed intake, $\mathrm{kg}$ & $3.12^{\mathrm{a}}$ & $3.07^{\mathrm{a}}$ & $1.69^{\mathrm{b}}$ & $1.83^{\mathrm{b}}$ & 0.06 & $<0.001$ & 0.59 & 0.22 \\
\hline Feed to gain ratio & $3.66^{\mathrm{b}}$ & $3.19^{\mathrm{b}}$ & $5.62^{\mathrm{a}}$ & $5.79^{\mathrm{a}}$ & 0.49 & 0.001 & 0.80 & 0.61 \\
\hline
\end{tabular}

$a, b, c$ Mean values with unlike superscript letters were significantly different $(P<0.05)$ $\mathrm{GB}$ diets, Chinese conventional diets; $\mathrm{B} \times \mathrm{D}$, breed $\times$ diet interaction 
Table 4 Effects of dietary protein/energy ratio and breed on carcass performance in pigs

\begin{tabular}{|c|c|c|c|c|c|c|c|c|}
\hline \multirow[t]{2}{*}{ Item } & \multicolumn{2}{|c|}{ Landrace pig } & \multicolumn{2}{|c|}{ Bama mini-pig } & \multirow[t]{2}{*}{ SEM } & \multicolumn{3}{|c|}{$P$-value } \\
\hline & GB diets & NRC diets & GB diets & NRC diets & & Breed & Diet & $B \times D$ \\
\hline \multicolumn{9}{|l|}{ Nursery phase } \\
\hline Pre-slaughter BW, kg & $19.39^{\mathrm{a}}$ & $19.15^{\mathrm{a}}$ & $12.00^{\mathrm{b}}$ & $14.40^{\mathrm{b}}$ & 0.84 & $<0.001$ & 0.21 & 0.13 \\
\hline Left carcass BW, kg & $6.25^{\mathrm{a}}$ & $6.17^{\mathrm{a}}$ & $3.20^{c}$ & $4.19^{b}$ & 0.32 & $<0.001$ & 0.16 & 0.10 \\
\hline Dressing percentage, $\%$ & $64.29^{\mathrm{a}}$ & $64.15^{\mathrm{a}}$ & $53.26^{\mathrm{C}}$ & $58.16^{b}$ & 1.32 & $<0.001$ & 0.08 & 0.06 \\
\hline Carcass length (bevel), $\mathrm{cm}$ & $25.69^{a}$ & $23.76^{\mathrm{a}}$ & $14.92^{\mathrm{b}}$ & $15.69^{b}$ & 1.13 & $<0.001$ & 0.61 & 0.24 \\
\hline Carcass length (straight), $\mathrm{cm}$ & $24.04^{\mathrm{a}}$ & $21.72^{\mathrm{a}}$ & $13.56^{\mathrm{b}}$ & $14.35^{\mathrm{b}}$ & 1.03 & $<0.001$ & 0.46 & 0.14 \\
\hline Backfat thickness, mm & $7.78^{\mathrm{a}}$ & $5.64^{\mathrm{b}}$ & $6.81^{\mathrm{ab}}$ & $7.19^{\mathrm{ab}}$ & 0.52 & 0.75 & 0.78 & 0.14 \\
\hline Loin-eye area, $\mathrm{mm}^{2}$ & $904.40^{\mathrm{b}}$ & $1,124.20^{a}$ & $372.30^{c}$ & $437.80^{c}$ & 73.41 & $<0.001$ & 0.06 & 0.30 \\
\hline Total skeletal muscle, $\%$ & $61.23^{\mathrm{a}}$ & $65.75^{\mathrm{a}}$ & $47.70^{b}$ & $46.38^{b}$ & 1.58 & $<0.001$ & 0.32 & 0.07 \\
\hline Total fat, \% & $6.47^{b}$ & $3.63^{\mathrm{b}}$ & $19.36^{\mathrm{a}}$ & $23.54^{\mathrm{a}}$ & 1.56 & $<0.001$ & 0.67 & 0.03 \\
\hline \multicolumn{9}{|l|}{ Growing phase } \\
\hline Pre-slaughter BW, kg & $58.51^{a}$ & $49.21^{b}$ & $33.62^{c}$ & $34.36^{\mathrm{c}}$ & 1.87 & $<0.001$ & 0.05 & 0.02 \\
\hline Left carcass BW, kg & $20.98^{\mathrm{a}}$ & $17.66^{\mathrm{b}}$ & $10.91^{\mathrm{c}}$ & $11.23^{c}$ & 0.71 & $<0.001$ & 0.07 & 0.03 \\
\hline Dressing percentage, $\%$ & $71.67^{\mathrm{a}}$ & $71.74^{\mathrm{a}}$ & $65.04^{b}$ & $65.13^{b}$ & 0.97 & $<0.001$ & 0.94 & 0.99 \\
\hline Carcass length (bevel), $\mathrm{cm}$ & $85.21^{\mathrm{a}}$ & $80.57^{b}$ & $69.33^{c}$ & $69.29^{c}$ & 1.18 & $<0.001$ & 0.08 & 0.08 \\
\hline Carcass length (straight), $\mathrm{cm}$ & $82.07^{\mathrm{a}}$ & $76.57^{b}$ & $59.00^{c}$ & $61.29^{c}$ & 1.12 & $<0.001$ & 0.20 & 0.004 \\
\hline Backfat thickness, mm & $6.07^{b}$ & $6.54^{\mathrm{b}}$ & $30.70^{\mathrm{a}}$ & $33.36^{\mathrm{a}}$ & 1.33 & $<0.001$ & 0.29 & 0.46 \\
\hline Loin-eye area, mm² & $1,919.70^{\mathrm{a}}$ & $1,724.20^{\mathrm{a}}$ & $696.70^{\mathrm{b}}$ & $671.70^{b}$ & 120.20 & $<0.001$ & 0.41 & 0.52 \\
\hline Total skeletal muscle, \% & $66.53^{\mathrm{a}}$ & $62.97^{b}$ & $40.70^{c}$ & $40.08^{c}$ & 1.08 & $<0.001$ & 0.09 & 0.22 \\
\hline Total fat, \% & $10.07^{c}$ & $13.45^{\mathrm{b}}$ & $34.60^{\mathrm{a}}$ & $35.88^{\mathrm{a}}$ & 0.87 & $<0.001$ & 0.02 & 0.28 \\
\hline \multicolumn{9}{|l|}{ Finishing phase } \\
\hline Pre-slaughter BW, kg & $86.79^{\mathrm{a}}$ & $92.03^{\mathrm{a}}$ & $49.84^{b}$ & $49.19^{b}$ & 2.15 & $<0.001$ & 0.36 & 0.24 \\
\hline Left carcass BW, kg & $32.39^{a}$ & $34.79^{a}$ & $17.14^{b}$ & $17.69^{b}$ & 0.90 & $<0.001$ & 0.16 & 0.38 \\
\hline Dressing percentage, $\%$ & $74.50^{\mathrm{a}}$ & $75.61^{\mathrm{a}}$ & $68.91^{c}$ & $71.90^{b}$ & 0.76 & $<0.001$ & 0.03 & 0.29 \\
\hline Carcass length (bevel), $\mathrm{cm}$ & $92.29^{a}$ & $92.00^{\mathrm{a}}$ & $81.80^{b}$ & $78.86^{\mathrm{b}}$ & 1.32 & $<0.001$ & 0.30 & 0.39 \\
\hline Carcass length (straight), $\mathrm{cm}$ & $87.40^{\mathrm{a}}$ & $87.83^{\mathrm{a}}$ & $79.90^{b}$ & $77.00^{\mathrm{b}}$ & 1.22 & $<0.001$ & 0.39 & 0.25 \\
\hline Backfat thickness, mm & $22.69^{b}$ & $23.52^{b}$ & $40.40^{\mathrm{a}}$ & $43.29^{\mathrm{a}}$ & 1.42 & $<0.001$ & 0.27 & 0.53 \\
\hline Loin-eye area, $\mathrm{mm}^{2}$ & $3,303.90^{\mathrm{a}}$ & $2,836.60^{\mathrm{a}}$ & $1,018.50^{b}$ & $812.40^{\mathrm{b}}$ & 155.78 & $<0.001$ & 0.07 & 0.47 \\
\hline Total skeletal muscle, $\%$ & $61.84^{\mathrm{a}}$ & $62.73^{\mathrm{a}}$ & $37.40^{b}$ & $38.38^{b}$ & 0.97 & $<0.001$ & 0.41 & 0.98 \\
\hline Total fat, $\%$ & $15.65^{\mathrm{b}}$ & $16.81^{\mathrm{b}}$ & $38.00^{\mathrm{a}}$ & $37.85^{\mathrm{a}}$ & 1.01 & $<0.001$ & 0.67 & 0.57 \\
\hline
\end{tabular}

a, b, c Mean values with unlike superscript letters were significantly different $(P<0.05) . n=8$

$\mathrm{GB}$ diets, Chinese conventional diets; $\mathrm{B} \times \mathrm{D}$, breed $\times$ diet interaction

Table 5 Effects of dietary protein/energy ratio and breed on meat quality in finishing pigs

\begin{tabular}{|c|c|c|c|c|c|c|c|c|}
\hline \multirow[t]{2}{*}{ Item } & \multicolumn{2}{|c|}{ Landrace pig } & \multicolumn{2}{|c|}{ Bama mini-pig } & \multirow[t]{2}{*}{ SEM } & \multicolumn{3}{|c|}{$P$-value } \\
\hline & GB diets & NRC diets & GB diets & NRC diets & & Breed & Diet & $B \times D$ \\
\hline pH value (45 min) & $6.38^{a}$ & $6.22^{\mathrm{ab}}$ & $6.08^{b}$ & $6.08^{b}$ & 0.07 & 0.01 & 0.34 & 0.36 \\
\hline $\mathrm{pH}$ value (24 h) & $5.45^{\mathrm{a}}$ & $5.49^{\mathrm{a}}$ & $5.29^{b}$ & $5.45^{\mathrm{a}}$ & 0.04 & 0.06 & 0.05 & 0.27 \\
\hline$L^{*}$ & 47.62 & 47.47 & 48.55 & 48.11 & 1.00 & 0.85 & 0.27 & 0.33 \\
\hline$a^{*}$ & $12.87^{c}$ & $13.80^{\mathrm{bc}}$ & $14.30^{\mathrm{ab}}$ & $15.29^{a}$ & 0.30 & $<0.001$ & 0.01 & 0.94 \\
\hline$b^{*}$ & 5.62 & 5.68 & 6.11 & 5.70 & 0.39 & 0.57 & 0.69 & 0.60 \\
\hline Drip loss, $\%$ & $1.99^{\mathrm{a}}$ & $1.53^{\mathrm{ab}}$ & $1.16^{\mathrm{b}}$ & $1.62^{\mathrm{ab}}$ & 0.20 & 0.12 & 0.99 & 0.05 \\
\hline Cooking yield, \% & 53.45 & 53.22 & 55.49 & 55.78 & 0.81 & 0.02 & 0.97 & 0.78 \\
\hline
\end{tabular}

$\mathrm{a}, \mathrm{b},{ }^{c}$ Mean values with unlike superscript letters were significantly different $(P<0.05) . n=8$

$\mathrm{GB}$ diets, Chinese conventional diets; $\mathrm{B} \times \mathrm{D}$, breed $\times$ diet interaction 
Table 6 Effects of dietary protein/energy ratio and breed on muscle chemical composition in pigs, \%

\begin{tabular}{|c|c|c|c|c|c|c|c|c|}
\hline \multirow[t]{2}{*}{ Item } & \multicolumn{2}{|c|}{ Landrace pig } & \multicolumn{2}{|c|}{ Bama mini-pig } & \multirow[t]{2}{*}{ SEM } & \multicolumn{3}{|c|}{$P$-value } \\
\hline & GB diets & NRC diets & GB diets & NRC diets & & Breed & Diet & $B \times D$ \\
\hline \multicolumn{9}{|l|}{ Nursery phase } \\
\hline \multicolumn{9}{|c|}{ Longissimus dorsi muscle } \\
\hline Dry matter & 23.75 & 23.68 & 23.61 & 24.09 & 0.33 & 0.69 & 0.55 & 0.41 \\
\hline Intramuscular fat & $1.22^{\mathrm{b}}$ & $1.06^{\mathrm{b}}$ & $2.82^{a}$ & $3.54^{\mathrm{a}}$ & 0.26 & $<0.001$ & 0.30 & 0.10 \\
\hline Crude protein & $18.34^{b}$ & $19.69^{a}$ & $19.47^{\mathrm{a}}$ & $19.61^{a}$ & 0.36 & 0.17 & 0.05 & 0.11 \\
\hline \multicolumn{9}{|l|}{ Biceps femoris muscle } \\
\hline Dry matter & $22.11^{b c}$ & $21.39^{c}$ & $23.39^{\mathrm{a}}$ & $23.15^{\mathrm{ab}}$ & 0.42 & 0.001 & 0.26 & 0.57 \\
\hline Intramuscular fat & $0.86^{c}$ & $0.99^{c}$ & $3.25^{a}$ & $2.45^{b}$ & 0.22 & $<0.001$ & 0.14 & 0.05 \\
\hline Crude protein & $17.60^{\mathrm{bc}}$ & $16.49^{c}$ & $18.51^{\mathrm{ab}}$ & $19.01^{\mathrm{a}}$ & 0.40 & $<0.001$ & 0.46 & 0.05 \\
\hline \multicolumn{9}{|l|}{ Psoas major muscle } \\
\hline Dry matter & 22.21 & 22.42 & 21.77 & 22.37 & 0.35 & 0.50 & 0.27 & 0.59 \\
\hline Intramuscular fat & $0.89^{b}$ & $0.94^{b}$ & $2.48^{\mathrm{a}}$ & $2.32^{a}$ & 0.20 & $<0.001$ & 0.78 & 0.62 \\
\hline Crude protein & $16.94^{\mathrm{b}}$ & $17.41^{\mathrm{b}}$ & $17.72^{\mathrm{ab}}$ & $18.56^{\mathrm{a}}$ & 0.35 & 0.01 & 0.09 & 0.63 \\
\hline \multicolumn{9}{|l|}{ Growing phase } \\
\hline \multicolumn{9}{|c|}{ Longissimus dorsi muscle } \\
\hline Dry matter & $25.07^{b}$ & $25.05^{b}$ & $29.11^{a}$ & $27.37^{\mathrm{a}}$ & 0.63 & $<0.001$ & 0.24 & 0.25 \\
\hline Intramuscular fat & $1.16^{\mathrm{b}}$ & $1.50^{\mathrm{b}}$ & $3.73^{\mathrm{a}}$ & $3.50^{\mathrm{a}}$ & 0.34 & $<0.001$ & 0.89 & 0.48 \\
\hline Crude protein & $21.27^{b}$ & $20.77^{b}$ & $21.98^{\mathrm{ab}}$ & $22.73^{\mathrm{a}}$ & 0.39 & 0.007 & 0.79 & 0.18 \\
\hline \multicolumn{9}{|l|}{ Biceps femoris muscle } \\
\hline Dry matter & $23.15^{b}$ & $22.89^{b}$ & $26.79^{a}$ & $23.76^{\mathrm{b}}$ & 0.75 & 0.01 & 0.05 & 0.10 \\
\hline Intramuscular fat & $1.01^{\mathrm{b}}$ & $0.81^{b}$ & $2.77^{\mathrm{a}}$ & $1.68^{\mathrm{b}}$ & 0.30 & $<0.001$ & 0.05 & 0.17 \\
\hline Crude protein & $19.80^{b}$ & $19.24^{b}$ & $22.13^{\mathrm{a}}$ & $19.35^{\mathrm{b}}$ & 0.71 & 0.13 & 0.04 & 0.16 \\
\hline \multicolumn{9}{|l|}{ Psoas major muscle } \\
\hline Dry matter & $23.67^{c}$ & $23.68^{\mathrm{c}}$ & $26.78^{a}$ & $24.97^{b}$ & 0.36 & $<0.001$ & 0.04 & 0.03 \\
\hline Intramuscular fat & $1.06^{\mathrm{b}}$ & $1.19^{b}$ & $4.66^{\mathrm{a}}$ & $2.25^{b}$ & 0.36 & $<0.001$ & 0.01 & 0.005 \\
\hline Crude protein & 19.82 & 20.17 & 21.07 & 20.84 & 0.51 & 0.10 & 0.91 & 0.62 \\
\hline \multicolumn{9}{|l|}{ Finishing phase } \\
\hline \multicolumn{9}{|c|}{ Longissimus dorsi muscle } \\
\hline Dry matter & $26.16^{c}$ & $26.56^{b c}$ & $28.74^{\mathrm{a}}$ & $28.26^{\mathrm{ab}}$ & 0.58 & 0.004 & 0.95 & 0.50 \\
\hline Intramuscular fat & $2.59^{\mathrm{b}}$ & $2.48^{\mathrm{b}}$ & $5.85^{\mathrm{a}}$ & $4.50^{\mathrm{a}}$ & 0.44 & $<0.001$ & 0.15 & 0.22 \\
\hline Crude protein & 21.32 & 22.02 & 21.73 & 21.93 & 0.47 & 0.76 & 0.40 & 0.64 \\
\hline \multicolumn{9}{|l|}{ Biceps femoris muscle } \\
\hline Dry matter & $24.97^{b}$ & $25.18^{b}$ & $25.43^{b}$ & $28.93^{\mathrm{a}}$ & 0.77 & 0.02 & 0.04 & 0.07 \\
\hline Intramuscular fat & $1.42^{c}$ & $1.88^{c}$ & $3.65^{b}$ & $5.23^{\mathrm{a}}$ & 0.31 & $<0.001$ & 0.01 & 0.12 \\
\hline Crude protein & $20.84^{\mathrm{ab}}$ & $21.19^{\mathrm{ab}}$ & $19.41^{b}$ & $22.85^{a}$ & 0.81 & 0.90 & 0.05 & 0.10 \\
\hline \multicolumn{9}{|l|}{ Psoas major muscle } \\
\hline Dry matter & 25.47 & 24.70 & 26.42 & 26.04 & 0.57 & 0.10 & 0.40 & 0.77 \\
\hline Intramuscular fat & $2.46^{\mathrm{b}}$ & $2.43^{b}$ & $4.01^{a}$ & $4.00^{\mathrm{a}}$ & 0.38 & 0.002 & 0.96 & 0.97 \\
\hline Crude protein & 21.08 & 21.30 & 20.70 & 20.91 & 0.48 & 0.51 & 0.71 & 0.99 \\
\hline
\end{tabular}

$a, b, c$ Mean values with unlike superscript letters were significantly different $(P<0.05) . n=8$

$G B$ diets, Chinese conventional diets; $B \times D$, breed $\times$ diet interaction

Chemical composition of muscle

Chemical compositions of LD, BF, and PM muscles were examined (Table 6). The lipid content of these muscles was significantly higher $(P<0.05)$ in Bama mini-pigs than in Landrace pigs. The DM contents of BF in the nursery phase; that of $\mathrm{LD}, \mathrm{BF}$, and $\mathrm{PM}$ in the growing 
Table 7 Effects of dietary protein/energy ratio and breed on the plasma biochemical parameters in pigs

\begin{tabular}{|c|c|c|c|c|c|c|c|c|}
\hline \multirow[t]{2}{*}{ Item } & \multicolumn{2}{|c|}{ Landrace } & \multicolumn{2}{|c|}{ Bama mini-pig } & \multirow[t]{2}{*}{ SEM } & \multicolumn{3}{|c|}{$P$-value } \\
\hline & GB diets & NRC diets & GB diets & NRC diets & & Breed & Diet & $B \times D$ \\
\hline \multicolumn{9}{|l|}{ Nursery phase } \\
\hline$A L P, U / L$ & $173.63^{b}$ & $148.88^{b}$ & $235.88^{\mathrm{a}}$ & $182.63^{\mathrm{ab}}$ & 18.64 & 0.01 & 0.04 & 0.45 \\
\hline GPT, U/L & 62.88 & 77.75 & 67.63 & 81.00 & 7.94 & 0.62 & 0.08 & 0.92 \\
\hline GOT, U/L & 64.00 & 89.00 & 71.50 & 101.63 & 14.75 & 0.50 & 0.07 & 0.86 \\
\hline $\mathrm{LDH}, \mathrm{U} / \mathrm{L}$ & 665.50 & 742.63 & 654.63 & 793.00 & 60.15 & 0.74 & 0.08 & 0.61 \\
\hline $\mathrm{CPK}, \mathrm{U} / \mathrm{L}$ & $1,622.00$ & $2,218.50$ & $1,654.40$ & $1,901.00$ & 347.96 & 0.68 & 0.23 & 0.62 \\
\hline GGT, U/L & 111.00 & 75.38 & 66.00 & 82.63 & 16.63 & 0.27 & 0.57 & 0.13 \\
\hline Alb, g/L & 31.53 & 32.31 & 34.97 & 34.74 & 2.14 & 0.18 & 0.89 & 0.81 \\
\hline $\mathrm{TP}, \mathrm{g} / \mathrm{L}$ & $61.92^{b}$ & $68.39^{\mathrm{ab}}$ & $64.67^{\mathrm{ab}}$ & $71.51^{a}$ & 2.72 & 0.29 & 0.02 & 0.94 \\
\hline AMM, $\mu \mathrm{mol} / \mathrm{L}$ & 213.31 & 113.75 & 167.10 & 129.14 & 34.25 & 0.66 & 0.05 & 0.38 \\
\hline $\mathrm{UN}, \mathrm{mmol} / \mathrm{L}$ & $6.79^{a}$ & $5.86^{\mathrm{ab}}$ & $5.06^{\mathrm{b}}$ & $5.81^{\mathrm{ab}}$ & 0.41 & 0.04 & 0.82 & 0.05 \\
\hline \multicolumn{9}{|l|}{ Growing phase } \\
\hline$A L P, U / L$ & $207.67^{\mathrm{a}}$ & $192.33^{\mathrm{a}}$ & $123.67^{\mathrm{b}}$ & $90.75^{\mathrm{b}}$ & 18.00 & $<0.001$ & 0.24 & 0.67 \\
\hline GPT, U/L & $99.67^{a}$ & $71.33^{b}$ & $69.50^{b}$ & $72.13^{b}$ & 4.76 & 0.03 & 0.31 & 0.17 \\
\hline GOT, U/L & 92.67 & 84.83 & 64.83 & 63.38 & 16.26 & 0.19 & 0.80 & 0.86 \\
\hline $\mathrm{LDH}, \mathrm{U} / \mathrm{L}$ & $854.21^{\mathrm{a}}$ & $996.22^{\mathrm{a}}$ & $490.50^{b}$ & $503.44^{b}$ & 116.31 & 0.003 & 0.56 & 0.62 \\
\hline $\mathrm{CPK}, \mathrm{U} / \mathrm{L}$ & $1,614.50$ & $1,640.00$ & $1,826.30$ & $1,608.00$ & 337.95 & 0.81 & 0.80 & 0.75 \\
\hline GGT, U/L & $120.00^{\mathrm{a}}$ & $71.00^{b}$ & $67.33^{b}$ & $72.00^{b}$ & 13.04 & 0.18 & 0.26 & 0.16 \\
\hline Alb, g/L & $48.98^{a}$ & $39.35^{b}$ & $38.65^{b}$ & $37.75^{b}$ & 1.73 & 0.005 & 0.01 & 0.03 \\
\hline $\mathrm{TP}, \mathrm{g} / \mathrm{L}$ & $87.28^{a}$ & $69.82^{b}$ & $84.80^{\mathrm{a}}$ & $85.02^{\mathrm{a}}$ & 3.52 & 0.12 & 0.04 & 0.03 \\
\hline $\mathrm{AMM}, \mu \mathrm{mol} / \mathrm{L}$ & 88.02 & 112.68 & 96.18 & 139.16 & 11.63 & 0.32 & 0.06 & 0.60 \\
\hline $\mathrm{UN}, \mathrm{mmol} / \mathrm{L}$ & 6.52 & 6.56 & 5.25 & 5.42 & 0.68 & 0.13 & 0.89 & 0.93 \\
\hline \multicolumn{9}{|l|}{ Finishing phase } \\
\hline$A L P, U / L$ & $114.00^{\mathrm{ab}}$ & $157.83^{\mathrm{a}}$ & $85.60^{b}$ & $117.14^{\mathrm{ab}}$ & 13.98 & 0.05 & 0.03 & 0.72 \\
\hline GPT, U/L & $62.80^{\mathrm{ab}}$ & $70.00^{\mathrm{a}}$ & $51.60^{\mathrm{b}}$ & $67.14^{\mathrm{ab}}$ & 4.70 & 0.22 & 0.05 & 0.46 \\
\hline GOT, U/L & 46.40 & 56.33 & 46.40 & 46.57 & 6.06 & 0.51 & 0.49 & 0.50 \\
\hline $\mathrm{LDH}, \mathrm{U} / \mathrm{L}$ & 498.40 & 551.33 & 377.80 & 387.43 & 49.59 & 0.03 & 0.60 & 0.72 \\
\hline CPK, U/L & $1,974.00^{\mathrm{a}}$ & $1,901.30^{\mathrm{a}}$ & $1,925.00^{\mathrm{a}}$ & $897.10^{b}$ & 267.43 & 0.11 & 0.10 & 0.15 \\
\hline GGT, U/L & 49.80 & 52.17 & 51.20 & 56.43 & 4.21 & 0.58 & 0.46 & 0.78 \\
\hline Alb, g/L & 39.30 & 40.30 & 37.50 & 39.17 & 1.26 & 0.34 & 0.38 & 0.82 \\
\hline $\mathrm{TP}, \mathrm{g} / \mathrm{L}$ & 75.88 & 76.67 & 82.12 & 81.94 & 2.60 & 0.08 & 0.92 & 0.88 \\
\hline AMM, $\mu \mathrm{mol} / \mathrm{L}$ & 88.98 & 97.60 & 72.64 & 87.01 & 11.87 & 0.35 & 0.43 & 0.84 \\
\hline $\mathrm{UN}, \mathrm{mmol} / \mathrm{L}$ & $5.12^{\mathrm{a}}$ & $4.03^{b}$ & $4.47^{\mathrm{ab}}$ & $5.29^{\mathrm{a}}$ & 0.28 & 0.18 & 0.38 & 0.05 \\
\hline
\end{tabular}

$\mathrm{a}, \mathrm{b}, \mathrm{c}$ Mean values with unlike superscript letters were significantly different $(P<0.05) . n=8$

GB diets, Chinese conventional diets; B $\times D$, breed $\times$ diet interaction; ALP, alkaline phosphatase; GPT, glutamic-pyruvic transaminase; GOT, glutamic-oxaloacetic transaminase; $\mathrm{LDH}$, lactate dehydrogenase; CPK, creatine phosphokinase; GGT, gamma-glutamyl transpeptidase; Alb, albumin; TP, total protein; AMM, blood ammonia; UN, urea nitrogen

phase; and that of LD and BF in the finishing phase were higher $(P<0.05)$ in Bama mini-pigs than in Landrace pigs. In addition, compared with the Landrace pigs, Bama mini-pigs had higher $(P<0.05) \mathrm{CP}$ levels in the $\mathrm{BF}$ and $\mathrm{PM}$ muscles in the nursery phase and in the LD muscle in the growing phase. Bama mini-pigs fed the GB diet had higher $(P<0.05)$ lipid content in the
BF muscle in the nursery phase, and significantly higher $(P<0.05)$ contents of DM, lipid, and CP in the $\mathrm{BF}$ and $\mathrm{PM}$ muscles in the growing phase, than those fed the NRC diet. In the finishing phase, Bama minipigs fed the GB diet had lower $(P<0.05)$ contents of $\mathrm{DM}$, lipid, and $\mathrm{CP}$ in the $\mathrm{BF}$ muscle than those fed the NRC diet. 


\section{Plasma metabolites}

Plasma biochemical analytes of Bama mini-pigs and Landrace pigs are listed in Table 7 . In the nursery phase, no breed $\times$ diet interactions were observed $(P>0.05)$ for plasma biochemical analytes except for UN $(P=0.05)$. For pigs fed the GB diet, the plasma ALP activity was lower $(P<0.05)$, but UN concentration was higher $(P<$ $0.05)$ in Landrace pigs than in Bama mini-pigs. Within each breed, diet did not influence $(P>0.05)$ the concentrations of plasma metabolites.

In the growing phase, breed $\times$ diet interactions were observed $(P<0.05)$ for plasma concentrations of TP and Alb. Plasma activities of ALP and LDH in Landrace pigs were higher $(P<0.05)$ than those in Bama mini-pigs. Landrace pigs fed the GB diet had higher $(P<0.05)$ plasma activities of GPT and GGT, and higher $(P<0.05)$ concentrations of TP and Alb than those fed the NRC diet.

In the finishing phase, no interactions were observed $(P>0.05)$ between breed and diet for any of the plasma biochemical analytes, except for $\mathrm{UN}(P=0.05)$. Landrace pigs fed the NRC diet had lower $(P<0.05)$ plasma UN concentration than those fed the GB diet. Bama minipigs fed the GB diet had higher $(P<0.05)$ plasma CPK activity than those fed the NRC diet.

\section{Discussion}

The growth performance of pigs and their meat quality depend on the interactive effects of genotype, rearing conditions, pre-slaughter handling, and carcass/meat processing [15-17]. The present study focused on evaluating the effects of breed and dietary protein/energy ratio on growth performance, carcass composition, and meat quality. We found significant interaction effects of breed and diet on growth performance and carcass composition. Bama minipigs grew more slowly than Landrace pigs, and their carcasses were composed of less lean meat and more fat than those of Landrace pigs. These findings indicate that there are obvious differences in carcass composition between breeds, as previously reported [18, 19]. Furthermore, the backfat in the native breed was much thicker than that in the Landrace breed. This was expected, as Landrace pigs are the result of several years of genetic selection through Mendelian genetics and molecular genetics approaches for traits including increased growth rate and reduced fat content [20]. Within each breed, pigs fed the NRC diet had considerably higher dressing percentage than those fed the GB diet. However, in Landrace pigs, GB diet promoted carcass length and lean percentage, especially during the growing phase. The accelerated development of bones and muscle may have resulted from compensatory growth due to low nutrition. Landrace pigs fed the NRC diet deposited less fat than Bama mini-pigs. This may result from the high capacity for muscle growth of fast-growing genotypes, such as Landrace pig, which can utilize high nutrient diets without increased lipid deposition.

Meat quality is one of the most important economic traits of farm animals, and it determines the suitability of meat for further processing and storage, including retail display. The main desirable attributes are $\mathrm{pH}$, color, drip loss, fat content, and composition [21]. In our study, Bama mini-pigs exhibited more carcass fat, higher IMF content, and greater backfat thickness, but reduced dressing percentage, lean content of carcass, drip loss, and LD muscle area than did Landrace pigs in the same phase. These findings agree with previous reports of the superior quality of local Chinese pigs [22, 23]. Several studies have shown different dietary protein/ energy ratios in animal feeds. Some of these studies examined the growth performance, body composition, and metabolism of aquatic livestock [24], and others were related to obesity and health of humans $[25,26]$. Dietary protein/energy ratio has a significant influence on the fat deposition and chemical composition of muscle. Barea et al. [27] reported that gain:feed and gain:metabolizable energy intake were improved by decreasing the ideal CP content of the diet. In their study, when a diet providing 95 g ideal CP per kg DM was fed, protein deposition reached a maximum value of $71 \mathrm{~g} /$ day. Hamill et al. [28] reported a two-fold increase in IMF content of the musculus semimembranosus of Duroc gilts fed a low-protein diet compared to those fed a high-protein diet. Moreover, they demonstrated, via transcriptome analysis, that regulation of IMF accumulation in response to dietary protein restriction is associated with modulation of gene pathways involved in lipid synthesis and degradation. A high IMF content, also called "marbling fat", is associated with improved eating quality of meat [29]. The threshold level of IMF in meat that results in a pleasing eating experience is $1.5 \%$ IMF, with $2-3 \%$ IMF considered necessary for optimum eating quality [30]. In our study, IMF contents of all muscle samples examined from Bama mini-pigs were greater than $1.5 \%$. However, the IMF contents varied across the different muscle samples, and IMF contents in BF of Bama mini-pigs differed across phases.

Activities of metabolic enzymes, including ALP, GPT, and GOT, in the blood change in response to the growth and development of animals [31]. Researchers have widely divided opinions on the relationship between ALP, GPT, and GOT and growth performance (especially for ADG) and carcass traits. ALP plays an important role in lipid metabolism; hence, increasing the plasma activity of this enzyme may be useful for promoting ADG [32]. GPT and GOT play a critical role in transamination and reflect the status of protein synthesis and catabolism [33]. An increase in the plasma activities of these enzymes can improve amino acid metabolism. LDH catalyzes the reversible transformation of pyruvate to lactate and plays a principal 
role in anaerobic cellular metabolism [34]. GGT catalyzes the transfer of the gamma-glutamyl group from glutathione to acceptor amino acids. In this study, plasma activities of ALP and LDH were higher in Landrace pigs than in Bama mini-pigs, especially in the growing phase. Plasma activities of GPT and GGT in Landrace pigs fed the GB diet were higher than those fed the NRC diet. All of these findings were coincident with growth performance within the same phase.

Nitrogen is an indicator of protein status [35] and has been used to determine protein requirements and lean tissue growth rates in pigs. Blood UN, as the ultimate and major nitrogenous product of protein and amino acid catabolism, is synthesized in the body via the ornithine cycle $[36,37]$. Plasma UN concentration reflects the balance status of amino acids, and is often used as an indicator of kidney and liver function, as well as an indicator of relative hydration status in animals. Low blood $\mathrm{UN}$ indicates a good balance of amino acids, and suggests relatively low urea synthesis and hydration in the liver and relatively high dietary protein efficiency [38]. In the present study, we found that Landrace pigs fed the NRC diet had lower plasma concentration of UN, whereas Bama mini-pigs fed the same diet had higher plasma concentration of UN, than did those fed the GB diet. These findings suggest an interaction effect between breed and diet. In addition, increased protein consumption by mammals results in increased fecal ammonia, which is a polluting substance $[39,40]$. Therefore, limiting protein ingestion will also limit the ammonia excretion in fecal substances; lower plasma concentrations of UN could decrease the emission of ammonia from pig production, thereby reducing environmental pollution.

\section{Conclusions}

Compared to Landrace pigs, Bama mini-pigs showed slower growth and lower carcass performance, but had better meat quality, which confirms that this breed of pig is well suited for the production of high-quality pork. Although dietary protein/energy ratio affects the growth performance of Landrace pigs, which depends on the growth stage, we found that the dietary protein/energy ratio did not affect Bama mini-pigs. This finding may be useful for reducing the feed cost and minimizing the adverse effects of ammonia release to the environment in indigenous pig production.

\section{Additional file}

Additional file 1: Table S1. Ingredients and nutrient levels of

experimental diets. (DOC $47 \mathrm{~kb}$ )

\section{Abbreviations}

ADFl: Average daily feed intake; ADG: Average daily gain; BF: Biceps femoris; CP: Crude protein; DM: Dry matter; F/G: Ratio of feed intake to body gain; IMF: Intramuscular fat; LD: Longissimus dorsi; PM: Psoas major.

\section{Competing interests}

The authors have declared that no competing interests exist.

\section{Authors' contributions}

YYL and XFK carried out the animal experiments and data analysis, and drafted the manuscript. FNL and YLY designed the study and revised the manuscript. GLJ and XX participated in the animal trial. BET, JPD, and XJY helped with data collection and analysis. All authors read and approved the final manuscript.

\section{Acknowledgements}

The present work was jointly supported by the National Basic Research Program of China (No. 2012CB124704 and 2013CB127305), and K.C. Wong Education Foundation, Hong Kong.

\section{Author details}

${ }^{1}$ Key Laboratory of Agro-ecological Processes in Subtropical Region, Hunan Provincial Engineering Research Center of Healthy Livestock and Poultry, and Scientific Observing and Experimental Station of Animal Nutrition and Feed Science in South-Central, Ministry of Agriculture, Institute of Subtropical Agriculture, Chinese Academy of Sciences, Changsha, Hunan 410125, China. ${ }^{2}$ Hunan Animal Science and Veterinary Medicine Research Institute, Changsha, Hunan 410131, China. ${ }^{3}$ College of Animal Science and Technology, Hunan Agricultural University, Changsha, Hunan 410128, China. ${ }^{4}$ Southern Research and Outreach Center, University of Minnesota, Waseca, MN 56093, USA. ${ }^{5}$ University of Chinese Academy of Sciences, Beijing 100049, China.

Received: 4 February 2015 Accepted: 21 July 2015

Published online: 15 August 2015

\section{References}

1. Dunshea FR, D'Souza DN, Pethick DW, Harper GS, Warner RD. Effects of dietary factors and other metabolic modifiers on quality and nutritional value of meat. Meat Sci. 2005;71(1):8-38.

2. Tan BE, Yin YL, Liu ZQ, Li XG, Xu HJ, Kong XF, et al. Dietary L-arginine supplementation increases muscle gain and reduces body fat mass in growing-finishing pigs. Amino Acids. 2009;37(1):169-75.

3. Kouba M, Sellier P. A review of the factors influencing the development of intermuscular adipose tissue in the growing pig. Meat Sci. 2011;88(2):213-20.

4. Kawaguchi H, Miyoshi N, Miura N, Fujiki M, Horiuchi M, Izumi Y, et al. Microminipig, a non-rodent experimental animal optimized for life science research: Novel atherosclerosis model induced by high fat and cholesterol diet. J Pharmacol Sci. 2011;115(2):115-21.

5. Mei J, Yin Z, Zhang J, Lui KW, Hu S, Peng Z, et al. A mini pig model for visualization of perforator flap by using angiography and MIMICS. Surg Radiol Anat. 2010;32(5):477-84.

6. Liu Y, Chen JY, Shang HT, Liu CE, Wang Y, Niu R, et al. Light microscopic, electron microscopic, and immunohistochemical comparison of bama minipig (sus scrofa domestica) and human skin. Comp Med. 2010;60(2):142-8.

7. Shang HT, Guo KA, Liu Y, Yang JD, Wei H. Constitutive expression of CYP3A mRNA in Bama miniature pig tissues. Gene. 2013;524(2):261-7.

8. Yin YL, Yao K, Liu ZJ, Gong M, Ruan Z, Deng D, et al. Supplementing L-leucine to a low-protein diet increases tissue protein synthesis in weanling pigs. Amino Acids. 2010;39(5):1477-86.

9. National Research Council (NRC). Nutrient requirements of swine. Washington, DC, USA: National Academy Press; 2012.

10. Ministry of Agriculture of the People's Republic of China. Feeding standard of swine (GB, NY/T 65-2004). Beijing, China: China Agriculture Press; 2004.

11. Kong XF, Wu GY, Liao YP, Hou ZP, Liu HJ, Yin FG, et al. Effects of Chinese herbal ultra-fine powder as a dietary additive on growth performance, serum metabolites and intestinal health in early-weaned piglets. Livest Sci. 2007;108(1-3):272-5.

12. Ministry of Agriculture of the People's Republic of China. Performance measurement technology regulations for Chinese lean pig. Beijing, China: China Standard Press; 1987.

13. Cunniff $P$ (Ed.). Official Methods of Analysis of AOAC International (16th edn, 3rd Rev). Association of Official Analytical Chemists, 1997, Gaithersburg, MD, USA. 
14. Kong XF, Wu GY, Liao YP, Houb ZP, Liu HJ, Yin FG, et al. Dietary supplementation with Chinese herbal ultra-fine powder enhances cellular and humoral immunity in early-weaned piglets. Livest Sci. 2007;108(1-3):94-8.

15. Teye GA, Sheard PR, Whittington FM, Nute GR, Stewart A, Wood JD. Influence of dietary oils and protein level on pork quality. 1. Effects on muscle fatty acid composition, carcass, meat and eating quality. Meat Sci. 2006;73(1):157-65

16. Hinson RB, Wiegand BR, Ritter MJ, Allee GL, Carr SN. Impact of dietary energy level and ractopamine on growth performance, carcass characteristics, and meat quality of finishing pigs. J Anim Sci. 2011;89(11):3572-9.

17. Suarez-Belloch J, Sanz MA, Joy M, Latorre MA. Impact of increasing dietary energy level during the finishing period on growth performance, pork quality and fatty acid profile in heavy pigs. Meat Sci. 2013;93(4):796-801.

18. Edwards DB, Bates RO, Osburn WN. Evaluation of Duroc- vs. Pietrain-sired pigs for carcass and meat quality measures. J Anim Sci. 2003;81(8):1895-9.

19. Renaudeau D, Mourot J. A comparison of carcass and meat quality characteristics of Creole and Large White pigs slaughtered at $90 \mathrm{~kg}$ BW. Meat Sci. 2007;76(1):165-71.

20. Suzuki K, Irie M, Kadowaki H, Shibata T, Kumagai M, Nishida A. Genetic parameter estimates of meat quality traits in Duroc pigs selected for average daily gain, longissimus muscle area, backfat thickness, and intramuscular fat content. J Anim Sci. 2005;83(9):2058-65.

21. Rosenvold K, Andersen HJ. Factors of significance, for pork quality - a review. Meat Sci. 2003;64(3):219-37.

22. Gil M, Delday MI, Gispert M, Furnols MFI, Maltin CM, Plastow GS, et al. Relationships between biochemical characteristics and meat quality of Longissimus thoracis and Semimembranosus muscles in five porcine lines. Meat Sci. 2008:80(3):927-33.

23. Lefaucheur L, Milan D, Ecolan P, Le Callennec C. Myosin heavy chain composition of different skeletal muscles in Large White and Meishan pigs. J Anim Sci. 2004;82(7):1931-41.

24. Mathis N, Feidt C, Brun-Bellut J. Influence of protein/energy ratio on carcass quality during the growing period of Eurasian perch (Perca fluviatilis). Aquaculture. 2003:217(1-4):453-64.

25. Noakes M, Keogh JB, Foster PR, Clifton PM. Effect of an energy-restricted, high-protein, low-fat diet relative to a conventional high-carbohydrate, low-fat diet on weight loss, body composition, nutritional status, and markers of cardiovascular health in obese women. Am J Clin Nutr. 2005;81(6):1298-306.

26. Millward DJ, Jackson AA. Protein/energy ratios of current diets in developed and developing countries compared with a safe protein/energy ratio: implications for recommended protein and amino acid intakes. Public Health Nutr. 2004;7(3):387-405.

27. Barea R, Nieto R, Aguilera JF. Effects of the dietary protein content and the feeding level on protein and energy metabolism in Iberian pigs growing from 50 to $100 \mathrm{~kg}$ body weight. Animal. 2007;1(3):357-65.

28. Hamill RM, Aslan O, Mullen AM, O'Doherty JV, McBryan J, Morris DG, et al. Transcriptome analysis of porcine M. semimembranosus divergent in intramuscular fat as a consequence of dietary protein restriction. BMC Genomics. 2013;14:14.

29. Wood JD, Nute GR, Richardson RI, Whittington FM, Southwood O, Plastow $\mathrm{G}$, et al. Effects of breed, diet and muscle on fat deposition and eating quality in pigs. Meat Sci. 2004;67(4):651-67.

30. Fortin A, Robertson WM, Tong AKW. The eating quality of Canadian pork and its relationship with intramuscular fat. Meat Sci. 2005;69(2):297-305.

31. Madrid J, Villodre C, Valera L, Orengo J, Martinez S, Lopez MJ, et al. Effect of crude glycerin on feed manufacturing, growth performance, plasma metabolites, and nutrient digestibility of growing-finishing pigs. J Anim Sci. 2013;91(8):3788-95.

32. Yang H, Fu Y, Chen AG. Relationship between blood biochemical indice and production performance in pigs. Animal science abroad. 2001;1:34-7.

33. Feng $L$, Peng $Y$, Wu P, Hu K, Jiang WD, Liu Y, et al. Threonine affects intestinal function, protein synthesis and gene expression of TOR in Jian Carp (Cyprinus carpio var. Jian). PLoS One. 2013;8(7), e69974.

34. Koukourakis Ml, Giatromanolaki A, Sivridis E, Bougioukas G, Didilis V, Gatter $\mathrm{KC}$, et al. Lactate dehydrogenase-5 (LDH-5) overexpression in non-small-cell lung cancer tissues is linked to tumour hypoxia, angiogenic factor production and poor prognosis. Br J Cancer. 2003;89(5):877-85.
35. Kohn RA, Dinneen MM, Russek-Cohen E. Using blood urea nitrogen to predict nitrogen excretion and efficiency of nitrogen utilization in cattle, sheep, goats, horses, pigs, and rats. J Anim Sci. 2005;83(4):879-89.

36. Lordelo MM, Gaspar AM, Bellego LL, Freire JP. Isoleucine and valine supplementation of a low-protein corn-wheat-soybean meal-based diet for piglets: growth performance and nitrogen balance. J Anim Sci. 2008;86:2936-41

37. Urschel KL, Shoveller AK, Uwiera RRE, Pencharz PB, Ball RO. Citrulline is an effective arginine precursor in enterally fed neonatal piglets. J Nutr. 2006;136:1806-13.

38. Hahn JD, Biehl RR, Baker DH. Ideal digestible lysine level for early-finishing and late-finishing swine. J Anim Sci. 1995;73(3):773-84.

39. Andriamihaja M, Davila AM, Eklou-Lawson M, Petit N, Delpal S, Allek F, et al. Colon luminal content and epithelial cell morphology are markedly modified in rats fed with a high-protein diet. Am J Physiol-Gastroint Liver Physiol. 2010;299(5):G1030-7.

40. Mouille B, Robert $\mathrm{V}$, Blachier F. Adaptative increase of ornithine production and decrease of ammonia metabolism in rat colonocytes after hyperproteic diet ingestion. Am J Physiol-Gastroint Liver Physiol. 2004;287(2):G344-51.

\section{Submit your next manuscript to BioMed Central and take full advantage of:}

- Convenient online submission

- Thorough peer review

- No space constraints or color figure charges

- Immediate publication on acceptance

- Inclusion in PubMed, CAS, Scopus and Google Scholar

- Research which is freely available for redistribution 